\title{
Challenges of Post ERCP Laparoscopic Cholecystectomy versus Elective Cholecystectomy
}

\author{
Ahmed M. Farrag, MD; Abd El Ghani El-Shamy, MD; Ahmed Kamal, MB.B.Ch \\ Department of Surgery, Faculty of Medicine, Ain Shams University, Egypt, Egypt
}

Background: Laparoscopic cholecystectomy (LC) post Endoscopic retrograde cholangio-pancreatography (ERCP) with endoscopic sphincterotomy (ES) is accepted as treatment of choice for choledocho-cholecystolithiasis. Studies have demonstrated that LC after ES is associated with difficulties, complications and higher conversion rate. Our study was to assess the challenges and complexities of LC after ERCP compared with standard elective LC for symptomatic uncomplicated cholecystolithiasis and assess the stenting effect when inserted on the following cholecystectomy and detect other factors that may cause post ERCP cholecystectomy challenges and complexities.

Patients and methods: Prospective controlled clinical trial was conducted over 50 patients : (Group A 25 patients) who had undergone a previous ERCP for choledocholithiasis (PES) and (Group B 25 patients) with cholecystolithiasis with no previous intervention before LC (NPES).

Results: Patients in PES group had higher risks for longer operative time (mean 36 min) which is statistically highly significant, the conversion rate in the PES group and the NPES group (12\% versus $0 \%$ ), were not statistically significant, duration of post-operative hospital stay in the PES group was longer than NPES group (statistically highly significant), there was more difficulty in achieving the critical view of safety in the PES group (easily achieved in $52 \%$ ) than NPES group (easily achieved in $92 \%$ ) (Statistically different), the amount of post-operative drain was higher in the PES group (30-300 ml sero-sanginous fluid) than the NPES group (15-30 ml serosanginous fluid) (statistically highly significant).

Conclusion: Laparoscopic cholecystectomy post ERCP especially when delayed or stent was inserted is a challenge for any surgeon with higher complexity and longer operative time and more conversions to open cholecystectomies with more difficulty to achieve critical view of safety. So, it has to be done by an experienced surgeon and rendezvous ERCP with laparoscopic cholecystectomy in same setting is advised with further prospective studies is needed with proper timing of interventions.

Key words: ERCP, cholecysto-lithisasis, sphincterotomy, stent, CBD.

\section{Introduction}

Endoscopic retrograde cholangio-pancreatography (ERCP) is indicated patients who have clinical features and radiologic evidence of CBD stones. ${ }^{1}$

Elective laparoscopic cholecystectomy (ELC) has risk of more complications after ERCP, with longer operative times, increased bleeding insults, and with higher conversion rate to open cholecystectomy, cause for these risks and complications is not fully understood, and these may be attributed to the severity of gallstone disease or because of ERCP. ${ }^{2}$

Previous studies have shown that LC after ES is more difficult than LC for uncomplicated cholelithiasis. ${ }^{3}$

ERCP itself may cause harm to structures in the hepato-duodenal ligament either during instrumentation and dissection of the biliary tract or as a direct contrast effect that causes peri-portal inflammation and fibrosis. ${ }^{3}$

The conversion rate after a previous ES has been reported to be as high as $8-55 \%$ versus $5 \%$ in patients with uncomplicated disease. ${ }^{4}$

It may be due to disruption of sphincter of Oddi and bacterial colonization and translocation into biliary tract leading to inflammation and subsequent scarring of the hepatoduodenal ligament hindering dissection of Calot's triangle and promote adhesions which is not easy to dissect, this theory of reflux and bacterial colonization is strengthened by the finding that bile in patients who have undergone a sphincterotomy during ERCP is colonized in about $60 \%$ of patients. ${ }^{5}$

LC is more difficult after ES it might be helpful to 
these patients to be operated by an experienced laparoscopic surgeon to decrease the risk of conversion and subsequent complications. ${ }^{6}$

The conversion rate is affected by the learning curve of surgeons and their skills. The surgeons performing LC during their learning curve are associated with a high conversion rate. ${ }^{7}$

\section{Aim of the work}

The study was to assess the challenges and complexities of LC after ERCP compared with elective LC for symptomatic uncomplicated cholecystolithiasis and assess the stenting effect when inserted on the following cholecystectomy and detect other factors that may cause post ERCP cholecystectomy challenges and complexities.

\section{Patients and methods}

This study was a prospective controlled clinical trial conducted at Ain Shams University Hospitals on two groups of patients 50 patients during period from June 2018 to June 2019 with follow up for 3 months. Group A (25 patients) patients who had undergone a previous ERCP for choledocholithiasis and Group B (25 patients) with cholecystolithiasis who had no previous intervention prior to LC.

We included any adult patient with cholecystolithiasis.

We excluded patients with previous abdominal surgery and patients with liver diseases and those with a history of biliary stricture, cholangitis, hepatopancreato-biliary malignancy, prior bile tree surgery, or prior PTC drainage.

Informed consent was taken from all patients accepted to participate in the study. Risks, complications and alternative procedures were explained to the patient. Confidentiality was assured of the personal data and medical information of all patients.

\section{Technique}

We standardize procedures in both groups

For Group A: Patients were prepared to LC within one month to 40 days after ERCP this is due to difficulties in admitting patients to operations as soon as possible due to waiting lists. The number of days between ERCP and LC (the interval) was counted from last ERCP (complete duct clearance or stent insertion after clearance) till the day of operation. Stenting is confined to patients either had stricture, stone passer, or delayed cholecystectomy after ERCP is highly suspected.

\section{Procedure}

The laparoscopic cholecystectomy in both groups was carried out using a standard four-trocar technique (Figure 1). Prophylactic antibiotic was not routinely administered. After achievement of critical view of safety cystic artery and duct were clipped and transected (Figures 2,3), the gallbladder was removed. Drain inserted in all operations of two groups for 24 hours to detect amount and nature of drained fluid. When indicated conversion was done by right subcostal incision.

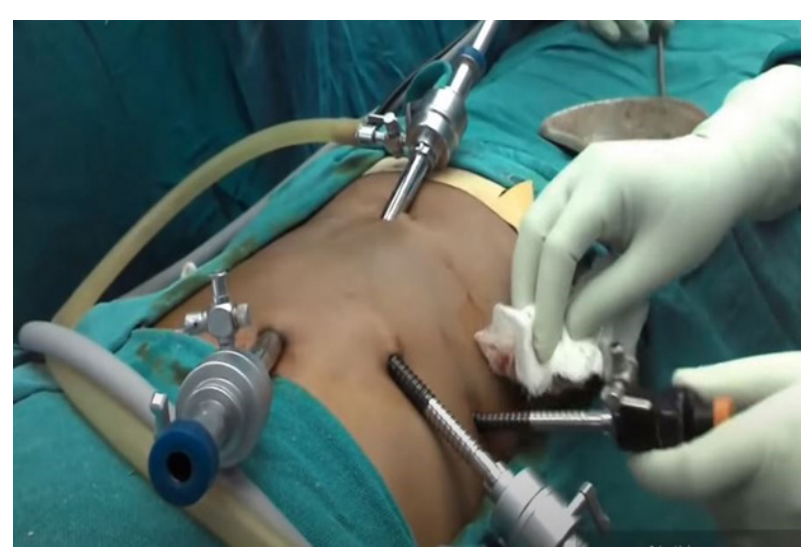

Fig 1: Standard four trocar technique for laparoscopic cholecystectomy.

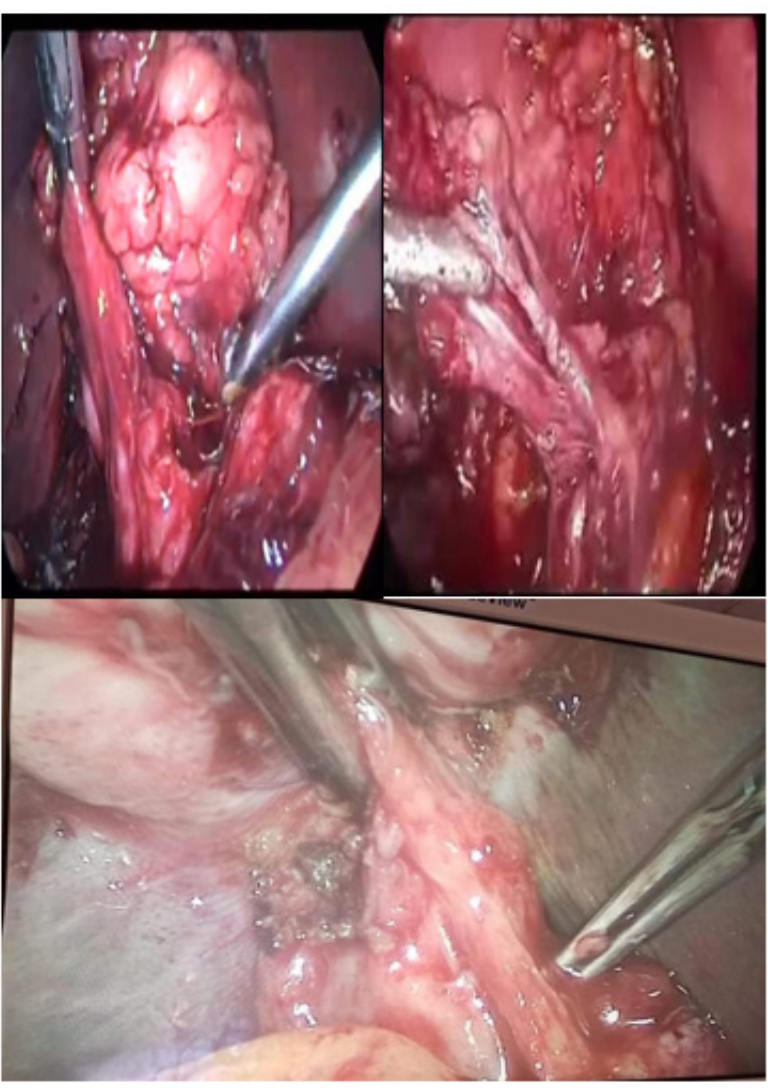

Fig 2: Difficulty in achieving critical view of safety due to severe adhesions in PES group. 


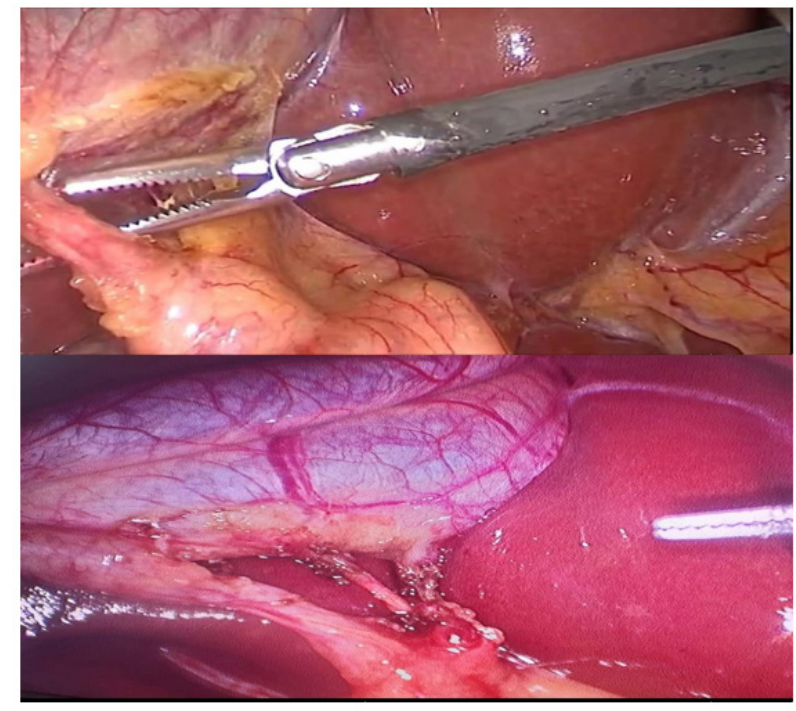

Fig 3: Easy acsess to critical view of safety in NPES group.

Outcome parameters and intra and postoperative assessment:

Length of the surgical procedure, conversion rate, uncontrollable bleeding, bile leakage during operations, difficulty in achieving critical view of safety, color and amount of drained fluid and post-operative hospital stay were recorded for all patients. All patients were followed for 3 months. Complications were recorded during hospital stay and outpatient clinic visits.

\section{Results}

Data management and analysis: Data were revised, coded, entered on a computer and analyzed using SPSS package version number 20 . Quantitative data were tested for normality with Shapiro-Wilk test and described as mean, standard deviation (SD) and range. Student t-test was used for comparing quantitative variables between two study group. Qualitative data were expressed as frequencies $(n)$ and percentage (\%). Fisher exact test was used to test the association between qualitative variables. P-value $\leq 0.05$ was considered significant.

25 patients actually underwent a cholecystectomy after ERCP, 12 patients were operated within 30 days, and 13 patients were operated from 30-40 days from ERCP. Successful ERCP was $100 \%$, all patients had CBD stones, and successful ductal clearance was done in 15 patients with no stent were inserted $(60 \%)$, the remaining 10 patients $(40 \%)$ underwent stenting after ductal clearance due to either stricture, stone passer, or delayed cholecystectomy after ERCP is highly suspected, post procedural pancreatitis developed in 1 patient (4\%). These data are shown in Table 1.

\section{Table 1: ERCP outcomes}

\begin{tabular}{lc}
\hline ERCP Parameter & No. (\%) \\
\hline Completion rate & $25(100)$ \\
CBD stones & $25(100)$ \\
Successful clearance with no stenting & $15(60)$ \\
Stented after ductal clearance & $10(40)$ \\
Post ES pancreatitis & $1(4)$ \\
\hline
\end{tabular}

Table 2: Patient characteristics

\begin{tabular}{lcccc}
\hline Patients & $\begin{array}{c}\text { PES } \\
(\mathbf{n = 2 5 )}\end{array}$ & $\begin{array}{c}\text { NPES } \\
(\mathbf{n = 2 5 )}\end{array}$ & P-value & Significance \\
\hline Age (years) & & & & \\
Mean (SD) & $37.92 \pm 8.93$ & $37.48 \pm 9.38$ & 0.866 & Non-significant \\
Male to female & $(1: 1.5)$ & $(1: 1.2)$ & 0.0128 & Non - significant \\
\hline
\end{tabular}

SD: Standard deviation.

PES: Previous ERCP.

NPES: No previous ERCP.

There was no significant difference in age between the PES and NPES groups. In the PES group. There was no difference in male to female ratio. 
Table 3: Procedural charactarestics

\begin{tabular}{lccccc}
\hline & PES $(\mathbf{n}=\mathbf{2 5})$ & NPES $(\mathbf{n}=\mathbf{2 5})$ & P-value & Significance \\
\hline $\begin{array}{l}\text { Length of procedure } \\
(\mathrm{min})\end{array}$ & Mean (range) & $89(60-120)$ & $53(42-70)$ & 0.000 & Highly significant \\
\hline
\end{tabular}

The mean length of LC was 36 min longer for the PES group compared with the NPES group

$(\mathrm{P}<0.001)$ which is highly significant.

Table 4: Conversion rate comparison in PES group and NPRS group

\begin{tabular}{lcccc}
\hline & PES $(\mathbf{n}=\mathbf{2 5})$ & NPES $(\mathbf{n}=\mathbf{2 5})$ & P-value & Significance \\
\hline conversion & $3(12 \%)$ & $0(0.0 \%)$ & 0.074 & Non -significant \\
\hline
\end{tabular}

There were three conversions in the PES group $(12 \%)$, in the NPES group there were zero

conversions (0\%) (Figure 4).

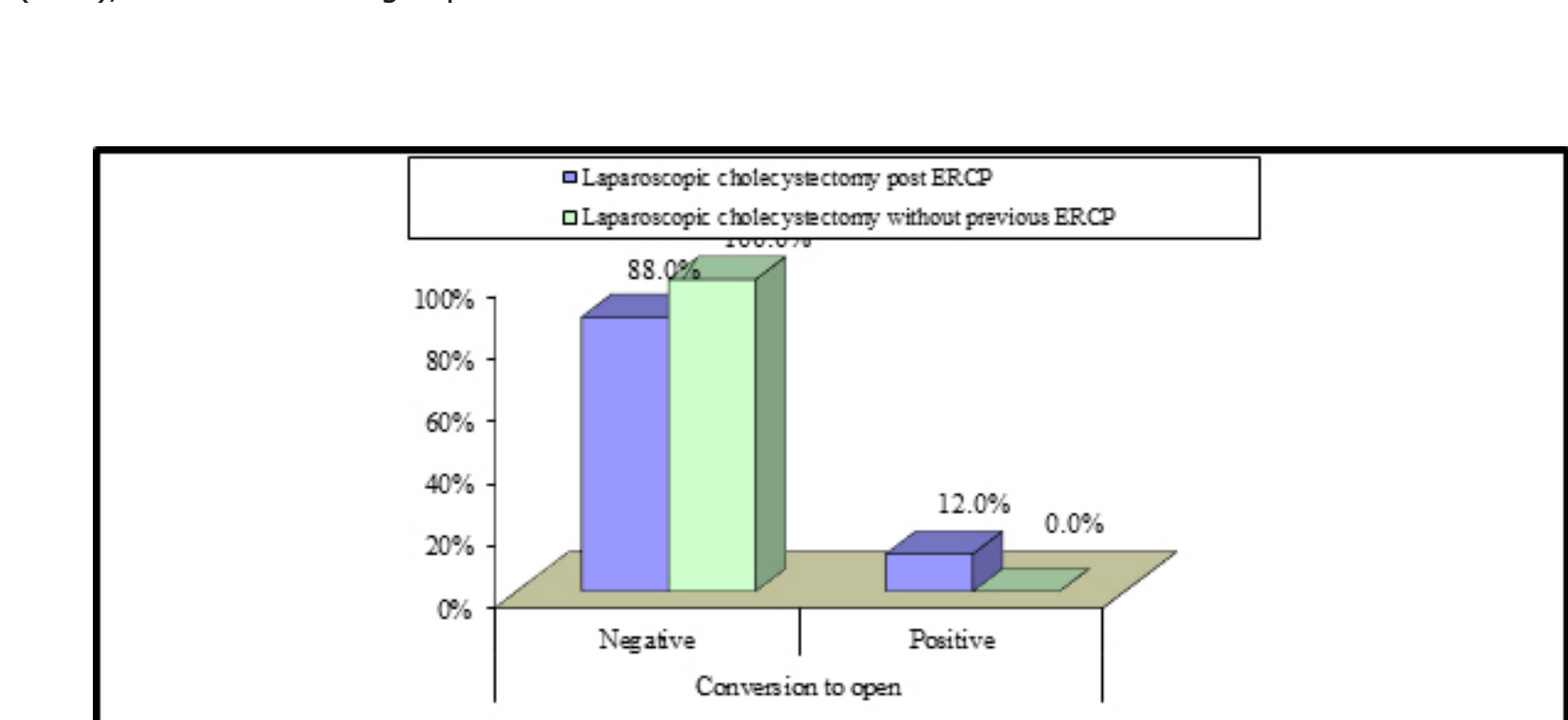

Fig 4: Chart shows conversion rate in the PES group and NPES group.

Table 5: Comparison between PES and NPES according to complications and post- operative hospital stay

\begin{tabular}{|c|c|c|c|c|c|}
\hline & & PES $(n=25)$ & NPES $(n=25)$ & P-value & Significance \\
\hline Complications & & $3(12 \%)$ & $2(8 \%)$ & 0.476 & Non -significant \\
\hline Post-operative hospital stay (days) & Mean (range) & $2(1-3)$ & $1(1-1)$ & 0.000 & Highly significant \\
\hline
\end{tabular}


Table 6: Comparison between PES and NPES according to Uncontrollable bleeding during operation

\begin{tabular}{lcccc}
\hline & PES $(\mathbf{n}=\mathbf{2 5})$ & NPES $(\mathbf{n}=\mathbf{2 5})$ & P-value & Significance \\
\hline Uncontrollable bleeding during operation & $3(12 \%)$ & $0(0.0 \%)$ & $(0.074)$ & Non-significant \\
\hline
\end{tabular}

During the operation, there was uncontrollable bleeding during operation from cystic artery in three patients in the PES group (12\%) due to severe adhesion and unclear anatomy, while there were no cases of uncontrollable bleeding in the NPES group $(0.0 \%)$.

Table 7: Comparison among both groups as regard amount of post-operative drained fluid

\begin{tabular}{lcccc}
\hline & PES $(\mathbf{n}=\mathbf{2 5})$ & NPES $(\mathbf{n}=\mathbf{2 5})$ & P-value & Significance \\
\hline $\begin{array}{l}\text { Amount of drained fluid } \\
\text { Mean (Range) }\end{array}$ & $80(30-300)$ & $20(15-30)$ & $<0.001$ & Highly significant \\
\hline
\end{tabular}

Amount of post-operative drained fluid is higher in the PES group (30-300 ml sero-sanguinous fluid) more than in the NPES group $(15-30 \mathrm{ml}$ serosanguinous fluid) ( $p$-value $<0.001$ ).

Table 8: Comparison between two groups as regard difficulty to achieve critical view of safety (CVS)

\begin{tabular}{lcccc}
\hline & PES $(\mathbf{n}=\mathbf{2 5})$ & NPES $(\mathbf{n}=\mathbf{2 5})$ & P-value & Significance \\
\hline $\begin{array}{l}\text { Number of patients with difficult to achieve } \\
\text { CVS }\end{array}$ & $12(48 \%)$ & $2(8 \%)$ & $<0.001$ & Highly significant \\
\hline
\end{tabular}

CVS: critical view of safety.

There were difficulty in achieving critical view of safety intraoperative in 12 cases $(48 \%)$ in the PES group, while there were difficulty in achieving critical view of safety in only 2 cases $(8 \%)$ in the NPES group ( $p$-value $<0,001$ ).

Table 9: Difficulty to achieve critical view of safety according to stent insertion among PES group

\begin{tabular}{lcccc}
\hline & PES with stent $(\mathbf{n = 1 0})$ & PES without stent $(\mathbf{n - 1 5})$ & P-value & Significance \\
\hline $\begin{array}{l}\text { Number of patient with } \\
\text { difficulty to achieve CVS }\end{array}$ & $8(70 \%)$ & $4(26 \%)$ & $<0.001$ & Highly significant \\
\hline
\end{tabular}

There was higher risk of difficulty in achieving critical view of safety in PES group with stent $(70 \%)$ more than others without stent $(26 \%)$ with statistically highly significant. as stent causes periductal fibrosis. 


\section{Discussion}

Data showed that patients who have undergone an ERCP for choledo-chocystolithiasis are more liable to difficult cholecystectomy, compared with patients with LC without ERCP.

Our study was conducted on two groups of patients undergoing LC. Although there were no statistically significant differences in rates of major complications, more challenges and complexity during cholecystectomy after ERCP were demonstrated.

LC after ERCP makes it difficult due to adhesions at the area of Calot's triangle, besides the risk of second-time anesthesia, ${ }^{7}$ more adhesions were found during LC after ERCP. ${ }^{2}$

Complications occurred in three patients from PES group (12\%) and in two patients in NPES group. 25 patients actually underwent a cholecystectomy after ERCP, 12 patients were operated within a month, and 13 patients were operated after 40 days. successful ERCP was $100 \%$, all patients had CBD stones, and successful ductal clearance was done in 15 patients (60\%), the remaining 10 patients (40\%) underwent stenting, post procedural pancreatitis developed in 1 patient (4\%).

No significant difference in age and sex between PES and NPES groups.

Although there was a higher conversion rate of LC after ERCP compared with LC without previous $E R C P$, but this difference fails to reach statistical significance $(P$-value $=0.074)$. There were three conversions in the PES group, two cases because of difficult visualization and difficulty to achieve critical view of safety with bleeding and one due to bleeding from short cystic artery. In the NPES group there were no conversions. Our study is in agreement with studies that showed a 6 fold higher conversion rate of LC after ERCP compared with LC for uncomplicated cholecystolithiasis. ${ }^{6}$

The conversion rate after a previous ES has been reported to be as high as $8-55 \%$ versus $5 \%$ in patients with LC without previous ERCP. Conversion to open cholecystectomy is associated with increased post-operative pain, pulmonary complication, longer hospital stay and slow recovery to normal daily activities. ${ }^{4}$

The conversion rate is affected by learning curve and skills of the surgeons. The surgeons performing LC during their learning curve are associated with a higher conversion rate.?

Amount of post-operative drain is more in PES group
$($ mean $=80 \mathrm{ml}$ ) than in NPES group (Mean $=20 \mathrm{ml}$ ) ( $p$-value $<0.001$ ) which is significantly statistically different. It could be attributed to minor bleeding from dissecting adhesions and use of saline wash to ensure good hemostasis especially in cases that converted to open surgeries and those with stent inserted.

Critical view of safety was hard to be achieved in 12 cases in the PES group, especially when stent was inserted, while it were hard to be achieved in only 2 cases in the NPES group ( $p$-value $<0,001$ ) which is of high statistical significance. There was difficulty in achieving it in PES group with stent $(70 \%)$ more than others without stent (26\%) which is of high statistical significance.

The mean length of LC was 36 min longer for the PES group compared with the NPES group $(P<0.001)$ which is highly significant. Long duration of the operation could be attributed to complexity of the procedure and severe adhesions in the PES group.

The median operative time in NPES group was $53 \mathrm{~min}$ and in PES group was $89 \mathrm{~min},(P<0.001)$ and it is statistically significant and reflects the complexity of procedure post ERCP. It is agreed with a study that showed significant longer duration of LC in PES group in comparison with NPES group. ${ }^{6}$

In our study, there was longer hospital stay in PES group as there more patients in PES were converted to open cholecystectomy which is statistically different, longer anesthesia time, and more postoperative complications such as wound infection and post-operative pain and chest infection and this is consistent with the results of other studies, ${ }^{4,6}$ But in contrast other study showed that there was no difference in hospital stay between the two groups. ${ }^{8}$

In our study there were three cases of uncontrollable bleeding while exploring the calot's triangle due to injury of cystic artery and failure to control bleeding with clips in the PES group, in addition to unclear anatomy so the decision for conversion was taken, while no cases were converted in the NPES group although lower cases but failed to reach statistical significance. Various factors such as improper technique and handling instruments and inability to recognize the anatomy contribute to occurrence of bleeding. ${ }^{9}$

Dissection during LC, especially the Calot's triangle, can lead to bleeding if the right hepatic artery or the portal vein is injured. This can also happen when the anatomy is distorted or unrecognized, and when there is persistence in using sharp dissection in a difficult Calot's, leading to bleeding and because of blind attempts to control the bleeder. Not being 
able to recognize the extent of injury and delaying conversion in such a situation definitely contributes to increasing the morbidity and mortality of the procedure. ${ }^{10}$

In our study patients were admitted within 40 days after ERCP this is due to waiting lists which may delay operative admissions for cholecystectomy after ERCP. We are convinced that early cholecystectomy after ERCP is safer and less liable for fibrosis and complications. It is also supported with another study that shows that early cholecystectomy after ERCP within 72 hours is safer. ${ }^{11}$ Delay of admissions for operations could be a confounder and affects the outcomes. So, single-stage management might be considered as the preferred approach but due to the existence of heterogeneity as patient's condition and operator's experience these findings should be taken into account in making treatment decisions.

This is supported with a study that demonstrated that after ERCP Culture growth was significantly higher, and fibrosis/collagen deposition in the gallbladder wall with injury to the mucosal epithelium was significantly more frequently detected by histopathological examination in the moderate and late period LC groups (moderate; $72 \mathrm{~h}-6$ weeks, and delayed; 6-8 weeks) than in the early period LC group (within 72 hours) $(p<0.05)$. Collagen deposition and damage to the mucosal epithelium were detected more in the moderate and delayed period LC groups than in the early period LC group. So, over a period of time, inflammation and the use of a contrast agent may increase the formation of fibrosis after ERCP. ${ }^{11}$

\section{Conclusion}

Laparoscopic cholecystectomy post ERCP especially when delayed or stent was inserted is a challenge for any surgeon with higher complexity and longer operative time and more conversions to open cholecystectomies with more difficulty to achieve critical view of safety. So, it has to be done by an experienced surgeon and rendezvous ERCP with laparoscopic cholecystectomy in same setting is advised with further prospective studies is needed with proper timing of interventions.

\section{References}

1. Paterson-Brown S: Core Topics in General and Emergency Surgery. A Companion to Specialist Surgical Practice. $4^{\text {th }}$ ed Philadelphia, PA: Saunders. 2009.

2. Chandio A, Timmons S, Majeed A, Twomey A, Aftab F: Factors influencing the successful completion of laparoscopic cholecystectomy. JSLS. 2009; 13(4): 581-6.
3. Bostanci E, Ercan M, Ozer I, Teke Z, Parlak E, Akoglu M: Timing of elective laparoscopic chole-cystectomy after endoscopic retrograde cholangiopancreato-graphy with sphincterotomy: A prospective observational study of 308 patients. Langenbecks Arch Surg. 2010; 395: 661-666.

4. Salman B, Yılmaz U, Kerem M, Bedirli A, Sare M, Sakrak O, Tatlicioglu E: The timing of laparoscopic cholecystectomy after endoscopic retrograde cholangiopancreatography in cholelithiasis coexisting with choledocholithiasis. J Hepatobiliary Pancreat Surg. 2009; 16: 832-836.

5. Reinders JS, et al: Early laparoscopic cholecystectomy improves outcomes after endoscopic sphincterotomy for choledochocystolithiasis. Gastroenterology. 20120; 138: 2315-2320.

6. Ellen T, Bert van Ramshorst: Laparoscopic cholecystectomy is more difficult after a previous endoscopic retrograde cholangiography. $\boldsymbol{H P \boldsymbol { B }}$ (Oxford). 2013; 15(3): 230-234.

7. Hong DF, Xin Y, Chen DW: Comparison of laparoscopic cholecystectomy combined with intraoperative endoscopic sphincterotomy and laparoscopic exploration of the common bile duct for cholecystocholedocholithiasis. Surg Endosc. 2006; 20: 424-427.

8. Suvikapakornkul R, Sawit K, Panuwat L: Retrospective comparison of onestage versus sequential ERCP and laparoscopic cholecystectomy in patients with symptomatic gallstones and suspected common bile duct stones. Thai J Surg. 2005; 26: 17-21.

9. Lauper $M$, Krähenbühl $L, A$ nation's experience of bleeding complications during laparoscopy, Am J Surg. 2000; 180(1): 73-7.

10. Tzovaras G, Dervenis C, Vascular injuries in laparoscopic cholecystectomy: An underestimated problem. Dig Surg. 2006; 23(56): 370-4.

11. Mehmet Aziret, Kerem Karaman, Metin Ercan, Erdem Vargöl, Bilal Toka, Yusuf Arslan, et al: Early laparoscopic cholecystectomy is associated with less risk of complications after the removal of common bile duct stones by endoscopic retrograde cholangiopancreatography. Turk $\boldsymbol{J}$ Gastroenterol. 2019; 30(4): 336-344. 